\title{
Serum metabolite profiling of a 4-Nitroquinoline-1-oxide- induced experimental oral carcinogenesis model using gas chromatography-mass spectrometry
}

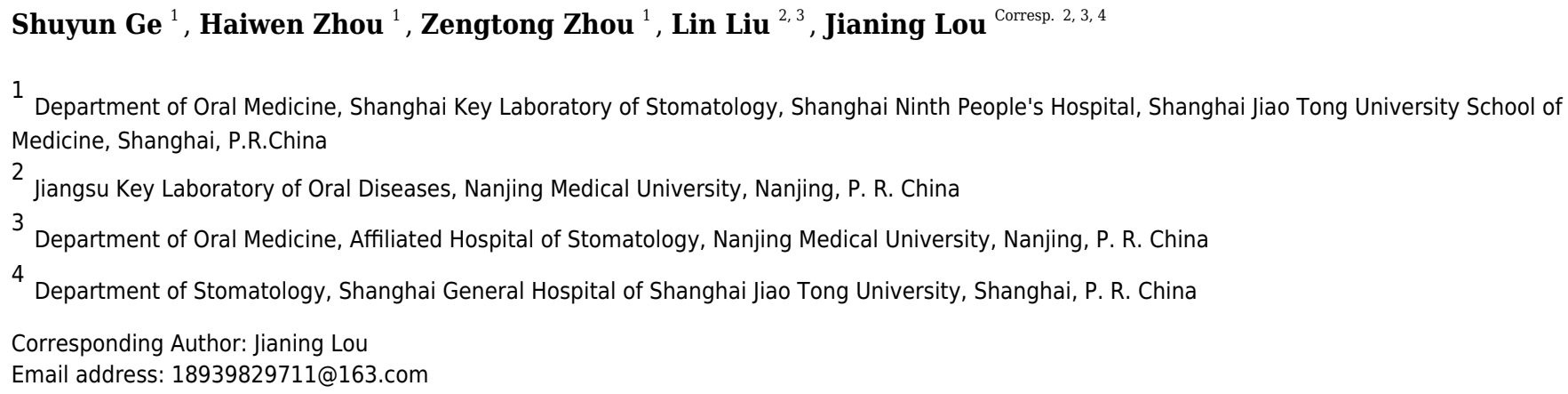

Background.Oral cancer progresses from hyperplastic epithelial lesions through dysplasia to invasive carcinoma. The critical needs in oral cancer treatment are expanding our knowledge of malignant tumour progression and the development of useful approaches to prevent dysplastic lesions. This study was designed to gain insights into the underlying metabolic transformations that occur during the process of oral carcinogenesis.

Methods.We used gas chromatography-mass spectrometry (GC-MS) in conjunction with multivariate statistical techniques to observe alterations in serum metabolites in a 4Nitroquinoline 1-oxide (4NQO)-induced rat tongue carcinogenesis model. Thirty-eight male rats were randomly divided into two groups, including the 4NQO-induced model group of 30 rats and the healthy control group of five rats. Animals were sacrificed at weeks 9, 13, 20, 24, and 32, post-4NQO treatment. Tissue samples were collected for histopathological examinations and blood samples were collected for metabolomic analysis. Partial least squares discriminate analysis (PLS-DA) models generated from GC-MS metabolic profile data showed robust discrimination from rats with oral premalignant and malignant lesions induced by 4NQO, and normal controls. Results. The results found 16 metabolites associated with 4NQO-induced rat tongue carcinogenesis. Dysregulated arachidonic acid, fatty acid, and glycine metabolism, as well as disturbed tricarboxylic acid (TCA) cycle and mitochondrial respiratory chains were observed in the animal model. The PLS-DA models of metabolomic results demonstrated good separations between the 4NQO-induced model group and the normal control group. Conclusion. We found several metabolites modulated by $4 \mathrm{NQO}$ and provide a good reference for further study of early diagnosis in oral cancer. 
1 Serum metabolite profiling of a 4-Nitroquinoline-1-

2 oxide-induced experimental oral carcinogenesis

3 model using gas chromatography-mass spectrometry

6 Shuyun $\mathrm{Ge}^{1}$, Haiwen Zhou ${ }^{1}$, Zengtong Zhou ${ }^{1}$, Lin Liu ${ }^{2,3}$, Jianing Lou ${ }^{2,3,4^{*}}$

$9{ }^{1}$ Department of Oral Medicine, Shanghai Key Laboratory of Stomatology, Shanghai Ninth 10 People's Hospital, Shanghai Jiao Tong University School of Medicine, Shanghai 200011, P.R.

11 China

12 '2Jiangsu Key Laboratory of Oral Diseases, Nanjing Medical University, Nanjing, P. R. China

13 32Department of Oral Medicine, Affiliated Hospital of Stomatology, Nanjing Medical University, 14 Nanjing, P. R. China

15 'Department of Stomatology, Shanghai General Hospital of Shanghai Jiao Tong University, 16 Shanghai 201620, P. R. China

19 Corresponding Author:

20 Jianing Lou

21 Department of Stomatology, Shanghai General Hospital of Shanghai Jiao Tong University,

22 Shanghai 201620, P. R. China

23 Email address:18939829711@163.com

24 


\section{Abstract}

26 Background.Oral cancer progresses from hyperplastic epithelial lesions through dysplasia to invasive carcinoma. The critical needs in oral cancer treatment are expanding our knowledge of malignant tumour progression and the development of useful approaches to prevent dysplastic lesions. This study was designed to gain insights into the underlying metabolic transformations that occur during the process of oral carcinogenesis.

Methods.We used gas chromatography-mass spectrometry (GC-MS) in conjunction with multivariate statistical techniques to observe alterations in serum metabolites in a 4-Nitroquinoline 1-oxide (4NQO)-induced rat tongue carcinogenesis model. Thirty-eight male rats were randomly divided into two groups, including the $4 \mathrm{NQO}$-induced model group of 30 rats and the healthy control group of five rats. Animals were sacrificed at weeks 9, 13, 20, 24, and 32, post-4NQO treatment. Tissue samples were collected for histopathological examinations and blood samples were collected for metabolomic analysis. Partial least squares discriminate analysis (PLS-DA) models generated from GC-MS metabolic profile data showed robust discrimination from rats with oral premalignant and malignant lesions induced by $4 \mathrm{NQO}$, and normal controls.

Results.The results found 16 metabolites associated with $4 \mathrm{NQO}$-induced rat tongue carcinogenesis. Dysregulated arachidonic acid, fatty acid, and glycine metabolism, as well as disturbed tricarboxylic acid (TCA) cycle and mitochondrial respiratory chains were observed in the animal model. The PLS-DA models of metabolomic results demonstrated good separations between the 4NQO-induced model group and the normal control group.

Conclusion. We found several metabolites modulated by $4 \mathrm{NQO}$ and provide a good reference for further study of early diagnosis in oral cancer.

Keywords: GC-MS; oral carcinogenesis; metabolomics; metabolites; tricarboxylic acid cycle 


\section{Introduction:}

50 Oral cancer is a type of malignant tumor with high degree of malignancy. Oral cancer accounts 51 for $7 \%$ of all new cancer cases worldwide, and 270,000 cases annually (Rivera, 2015). Over the 52 past several years, the incidence of oral cancer has increased, especially in developing countries 53 such as Sri Lanka, India, Pakistan and Bangladesh, where it accounts for up to $25 \%$ of all cancers(Krishna et al., 2013). Despite the development of surgery, radiotherapy, and chemotherapy in recent years, the five-year survival rate of oral cancer is about $50 \%$. Moreover, post-operative patients experience morphologic changes and different degrees of loss of function. Patients may experience significant dysfunction in talking, swallowing, with alteration of cosmetic appearance, and sensory impairment, as well as chronic pain. Additionally, some healed patients also relapse with a few years of treatment. All these factors when compounded lead to poor mental health.

Oral leukoplakia is acknowledged as a common precancerous lesion. The canceration rate of oral leukoplakia is $0.13 \%-34 \%($ Warnakulasuriya \& Ariyawardana, 2016). Oral leukoplakia is an important source of oral squamous cell carcinoma (OSCC). The World Health Organization

$64(\mathrm{WHO})$ has considered the control and prevention of oral premalignant lesions and oral cancer as one of the top urgent tasks in the"21st century global oral health strategy". Thus, screening biomarkers of malignant transformation of oral leukoplakia is important for preventing oral cancer. Metabolomics is a discipline focused on the changes to endogenous metabolites of biological systems following perturbation by a stimulus (such as a specific gene mutation or environmental change). The formation and development of tumors may be due to changes in the metabolic pathways of cells, which results in the loss of normal cell growth regulation and 71 abnormal proliferation. Metabolomics uses high-throughput, high sensitivity and high precision 72 instrumentation to dynamically analyze the changes in metabolites and identify cells as "cancer 73 precancerous - normal". Thus, Metabolomics detects the metabolic markers indicative of the 74 formation and development of cancer. The concentration of proteins, peptides, and metabolites regulating cell growth in multiple cancers were differentiated in tissue fluid than cells (Armitage \& 
76 Ciborowski, 2017, Kaushik \& DeBerardinis RJ, 2018). These minute changes in gene and protein expression are amplified at the metabolite level. Thus, in contrast to gene chip and proteomic approaches, metabolomics is more likely to detect biomarkers associated with cancer(Olivares et al., 2015, Armitage \& Ciborowski, 2017, Beger, 2013).

Using a high-resolution testing instrument (such as HR - MAS NMR, High-Resolution GCMS, High-Resolution LC-MS, et al.) and multivariate statistical analysis methods, metabolomics can accurately identify metabolites that change over the course of cancer development and can be used to evaluate tumors(Blekherman et al., 2011, Bathen et al., 2010, Wen et al., 2010). ${ }^{1}$ HNMR combined with partial least squares-discriminant analysis (PLS-DA) were used to identify differential metabolites among different groups which is refers to the different developmental stages of chemically induced oral cancer. This approach was used to determine that the levels of valine, lactic acid salt, alanine, and citric acid salt were reduced in oral cancer cells compared to healthy controls, whereas signals arising from glucose, pyruvate, acetone, acetoacetate, 3hydroxybutyrate and 2-hydroxybutyrate(Wu et al., 2010, Markuszewski et al., 2010, Kim et al., 2010). GC-MS has high sensitivity and high specificity, However, less applied to studies of oral cancer.

The 4-Nitroquinoline 1-oxide (4NQO)-induced carcinogenesis model is widely used for studies of oral cancer development and for screening cancer chemopreventive drugs. In this study, we utilized a gas chromatography-mass spectrometry (GC-MS)-based serum metabolite profiling approach to discriminate $4 \mathrm{NQO}$-induced oral carcinogenesis from normal controls at different times, and to monitor the changes in metabolites between such samples.

\section{Materials and methods:}

\section{NQO-induced rat carcinogenesis and sampling}

Rats were obtained from Shanghai Sippr-BK laboratory animal Co. Ltd. A total of 38 Wistar rats with origins in closed groups were used at 160 days old, and $220 \pm 10 \mathrm{~g}$ in body weight. Rats were fed full-value nutritional granulated fodder and raised in separate cages (four rats per cage). 
103 Rats were transferred to the holding room under controlled conditions, with a temperature of 23 $104 \pm 2^{\circ} \mathrm{C}$, and $30-50 \%$ humidity. $4 \mathrm{NQO}$ (Sigma, USA) was dissolved in distilled water to a

105 concentration of $0.002 \%$ and was placed in a brown bottle at $4^{\circ} \mathrm{C}$ until use. All animals and

106 experimental procedures were approved by the Management Committee of Animal Experimental

107 Ethical Shanghai Ninth People's Hospital affiliated to shanghai JiaoTong University, School of 108 Medicine HKDL (2016) 2.

109 The 38 rats were divided into two groups as follow. A: control group, given water $(n=5)$; $B$ :

110 model group, given 4NQO $(n=33)$

111 The criteria were established for euthanizing animals prior to the planned end of the 112 experiment. Rats were sacrificed at 9, 13, 20, 24, and 32 weeks from the beginning of the

113 experiment to collect the tissues Representing temporal carcinogenesis progression that 114 demonstrates multiple dysplastic, preneoplastic, and neoplastic lesions after long-term treatment.

115 The mice were sacrificed by intraperitoneal injection of sodium phenobarbital. Animal carcasses 116 were being loaded into the garbage bag and handled by Institute of Laboratory Animals. We

117 collected serum samples and stored it at $-80^{\circ} \mathrm{C}$ for GC-TOF mass spectrometry. Besides, we 118 collected the tissue samples for histopathological examination.

\section{Pathological examinations}

121 Gross lesions were identified and photographed. The histological determinations of squamous 122 neoplasia were performed by a pathologist on the sectioned tissue samples. The sections from 123 the tongues were deparaffinized, rehydrated, and stained with hematoxylin-eosin (HE) for 124 histopathological analyses. The lesions were classified into four types: mild epithelial dysplasia,

125 moderate epithelial dysplasia, severe epithelial dysplasia, and squamous cell carcinoma (SCC).

126 Epithelial lesions were diagnosed according to the criteria described by the WHO(Leininger \& 127 Jokinen, 1994)(Kramer et al., 1978). 
130 As the procedure we previously described, serum metabolites were analyzed by chemical 131 derivatization and slightly modified (Chen et al., 2011, Wei et al., 2012). We added $100 \mu \mathrm{L}$ aliquot

132 of serum to two internal standard solutions, $10 \mu \mathrm{L} \mathrm{L}$-2-chlorophenylalanine in water $(0.3 \mathrm{mg} / \mathrm{mL})$ 133 and $10 \mu \mathrm{L}$ heptadecanoic acid in methanol $(1 \mathrm{mg} / \mathrm{ml})$. Then, we swirled it for $10 \mathrm{~s}$. We extracted 134 a mixture with $300 \mu \mathrm{L}$ of methanol/chloroform (3:1) in eddy $30 \mathrm{~s}$. We stored the samples at $-20^{\circ} \mathrm{C}$ 135 for $10 \mathrm{~min}$ and centrifuged it at $12,000 \mathrm{~g}$ for $10 \mathrm{~min}$. Then, we transferred $300 \mu \mathrm{L}$ supernatant to 136 an aliquot for vacuum drying at room temperature. The residue was derived using a two-step 137 method. First, we added $80 \mu \mathrm{L}$ methoxyamine $(15 \mathrm{mg} / \mathrm{mL}$ in pyridine) to the bottle and incubated 138 it at $30^{\circ} \mathrm{C}$ for $90 \mathrm{~min}$. Then $80 \mu \mathrm{L}$ BSTFA (1\% TMCS) was added and incubated at $70^{\circ} \mathrm{C}$ for 60 $139 \min$.

We injected each $1 \mu \mathrm{L}$ derived solution into antae $6890 \mathrm{~N}$ gas chromatography in a non-

141 splitting manner in combination with Leco Pegasus IV time-of-flight MS instrument (Leco, St. 142 Joseph/MI, USA). Run alternately in control-model-control order to reduce system analysis bias. 143 Db-5ms capillary column (30 m × $250 \mu \mathrm{m}$ i.d., $0.25 \mu \mathrm{m}$ film thickness; (5\%-phenyl)-

144 methylpolysiloxane bonded and cross-linked; Agilent J\&W Scientific, Folsom, CA) was used for 145 separation. Helium was carrier gas with a constant flow rate of $1.0 \mathrm{~mL} / \mathrm{min}$. The injection 146 temperature was set at $270{ }^{\circ} \mathrm{C}$, the transfer interface temperature at $260{ }^{\circ} \mathrm{C}$, and the ion source 147 temperature at $200{ }^{\circ} \mathrm{C}$. We programmed GC temperature as $80^{\circ} \mathrm{C}$ isothermal heating for $2 \mathrm{~min}$

148 Then the oven temperature was rise to $180{ }^{\circ} \mathrm{C}$ by $10^{\circ} \mathrm{C} / \mathrm{min}, 240^{\circ} \mathrm{C}$ by $5^{\circ} \mathrm{C} / \mathrm{min}$, and $290^{\circ} \mathrm{C}$ by $14925^{\circ} \mathrm{C} / \mathrm{min}$. Finally, it was kept at $290^{\circ} \mathrm{C}$ for $9 \mathrm{~min}$. Electron shock ionization $(70 \mathrm{eV})$ in a full scan mode (M/Z30-600) was used to obtain 20 spectra /s in an MS setting.

\section{1}

\section{GC-MS data analysis:}

153 The data analyzed by GC-MS was converted to NetCDF format through the data analysis 154 interface of PE instrument (PerkinElmer Inc., USA). Subsequently, we extracted each file by a 155 custom script in MATLAB 7.0 (The MathWorks, Inc., USA) to perform a data preprocessor that 156 included baseline correction, peak deconvolution and calibration, exclusion of internal standard 
157 (I.S.) and solvent peaks, and normalization to the total ion current. The output data were organized 158 in the form of any arbitrary peak index (retention time-m/z pairs), sample names (observations),

159 and peak intensity information (variables). Internal criteria and any known artificial peaks, such 160 as those caused by noise, column bleeding, and ethyl chloroformate derivative processes, were 161 removed from the data set. Partial least-squares discriminant analyses (PLS-DA) were performed 162 by SIMCA-P 12.0 software (Umetrics, Umeå, Sweden). Based on a variable importance in 163 projection (VIP) threshold (VIP > 1) from the 7-fold cross-validated PLS-DA model, a number of 164 metabolites responsible for the differentiation of metabolic profiles of the 4NQO-induced model 165 group and the normal control group could be obtained. Meanwhile, through the parametric student $166 \mathrm{t}$ test and non-parametric Wilcox-Mann-Whitney test in the Matlab statistical toolbox, we verified

167 the metabolites identified by the PLS-DA model at the univariate level, and the critical value was 168 set at 0.05 . The corresponding fold changes showed the differences of these selectable 169 metabolites between the 4NQO-induced model group and the normal control group. Additionally, 170 compound identification was performed by comparing the mass fragments using the NIST 05

171 Standard mass spectral databases in NIST MS search 2.0 (NIST, Gaithersburg, MD) software 172 with a similarity of more than $70 \%$, verified using available citric acid reference compounds.

174 Results:

175 Histological changes of the mucosa following 4NQO administration

176 Rats showed no overt ill effects from the carcinogen applications. The body masses of control 177 and experimental animals remained similar until the end of the experiment, when the 4NQO178 treated animals tended to be lighter than the controls. Such findings were likely due to the 179 decreased food intake in rats with oral carcinomas.

180 The initial change of mild epithelial dysplasia was observed at 9 weeks following 4NQO 181 treatment. The thickness of the spinous cell layer gradually increased and occasionally exhibited 182 disordered basal cells. After 13 weeks post-4NQO treatment, further dysplastic changes occurred, 183 from moderate epithelial dysplasia, to severe epithelial dysplasia, and ultimately, invasive 
184 squamous cell carcinoma (SCC) being observed in the majority of animals (Figure 1A-E).

185 Histological changes in each group were shown in Table 1. Typical histopathological results of

186 oral lesions caused by 4NQO were shown in Figure 1.

187

\section{Metabolic profiles between the model group and controls}

189 First, we set the $Y$ value of normal group samples to 0 , while that of the model group was set to

190 1, join Y2 (sampling time point) and Y3 (pathological results). With the three guidance variables, 191 we established a PLS-DA model for multivariate statistical analysis. The separation trends and 192 sampling time points were closely linked, and thus, we observed the physiological changes of the 193 normal group. The result of the PLS-DA model revealed the separation of rats with SCC and 194 dysplasia from the normal group. The PLS-DA model demonstrated satisfactory modeling and 195 predictive abilities using one predictive component and two orthogonal components $(\mathrm{R} 2 \mathrm{X}=0.397$, $196 \mathrm{R} 2 \mathrm{Y}=0.982, \mathrm{Q} 2=0.762$ ), achieving a distinct separation between the metabolite profiles of the 197 4NQO-induced model group and normal control group (Figure 2).

198 We used MS spectrum database to identify 16 metabolites and confirmed the reference 199 standard between differential variables by using VIP values (VIP > 1) in the PLS-DA model and 200 the Wilcoxon-Mann-Whitney test $(p<0.05)$ (Table 2). Among the identified metabolites in the 201 model group, glycolysis showed the most significant change compared to controls. The altered 202 serum metabolites included elevated lactic acid, citric acid, pyruvate, arachidonic acid, glycine, 203 and decreased 3',5'-cyclic dGMP, PGF2alpha dimethyl amide, and 1-hexadecyl-2-acetyl-glycero204 3-phosphocholine in the model group, compared to normal controls.

206 Discussion:

207 4-Nitroquinoline 1-oxide-induced experimental oral carcinogenesis

208 Several animal models for oral SCC were previously generated, including those for hamsters, 209 rats, and mice.The 4NQO-induced tongue carcinogenesis model is anatomically more similar to 210 human SCC, more convenient, and likely more reproducible, as the carcinogen is administered 
211 in the drinking water. The use of $4 \mathrm{NQO}$ resulted in a transient model of cancer progression that

212 showed a variety of dysplasia, precancerous and neoplastic lesions after long-term treatment.

213 These continuously changing epithelial cells mimic the transformation of human oral tumors. In

214 addition, 4NQO-induced oral cancer in mice is similar to human head and neck squamous cell

215 carcinoma, affecting the expression of many genes involved in human tumorigenesis. Thus, this

216 animal model is widely used for studies of oral cancer development and to screen

217 chemopreventive drugs(Kanojia\& Vaidya, 2006).

218 4-Nitroquinoline 1-oxide is a water-soluble quinoline derivative that can cause DNA adduct

219 formation, resulting in adenosine substitution for guanosine. 4NQO also undergoes redox cycling

220 to produce reactive oxygen species that result in mutations and DNA strand breaks.

221

\section{Metabolite variations in the 4NQO-induced oral carcinogenesis model}

223 Metabolomics has great potential for the early detection and mechanisms of oral cancer. In our 224 study, the serum metabolome results of $4 \mathrm{NQO}$-induced tongue carcinogenesis at weeks 9,13 , 225 20, 24, and 32 showed progressive metabolic disturbance in the serum metabolome during 226 4NQO-induced oral carcinogenesis. Our study, based on the metabolomics of GC-MS, showed

22716 differentially expressed serum metabolites in the model group. The derived PLS-DA model 228 showed a good separation between the model group and the controls.

Arachidonic acid (ARA) is an n-6 essential fatty acid and also a major constituent of 230 biomembranes. ARA is released from membranes by phospholipase A2 and converted into 231 various lipid mediators that exert many physiological actions. ARA can be converted (1) by 232 cyclooxygenases (COXs) to prostaglandin E2 (PGE2) and thromboxane (TxA2); (2) by 233 mammalian lipoxygenases (LOXs) to four major hydroxyeicosatetraenoic acid (5-, 8-, 12-, and 234 15-HETE); and (3) by cytochrome P450 to epoxyeicosatrienoic acids (EETs) and HETEs. Many 235 studies have shown that lipid mediators derived from ARA, such as prostaglandin E2 (PGE2) and 236 leukotrienes, and the enzymes involved in their production, such as cyclooxygenases (COXs), 
237 lipoxygenases (LOXs), and cytochrome P450, are centrally involved in apoptosis and 238 angiogenesis.

239 The metabolism of ARA plays a role in the pathogenesis of many human cancers (Sakai 240 et al., 2012, Ninomiya et al., 2013, Yarla et al., 2016). Abnormally high levels of serum ARA during

241 the process of oral carcinogenesis suggests that a metabolic imbalance causes the high levels of 242 proliferative eicosanoids, such as PGE2, that are commonly found in tumor cells.

243 Lactic acid is the final product of the glycolysis pathway, which participates in energy

244 metabolism. The level of lactic acid and fructose in rats with SCC and dysplasia were elevated,

245 compared to the normal group. Lactic acid accumulation may be indicative of enhanced 246 glycolysis. Enhanced glycolysis is considered a common feature of tumors, which is called the 247 "Warburg effect" (WARBURG, 1956). Lactic acid levels were significantly elevated in head and 248 neck cancer, among others (Dart, 2016, Ohashi et al., 2017). In some cancers, pyruvate can be 249 converted into lactic acid under aerobic conditions (Choi et al., 2013). Fructose can also 250 participate in the glycolysis pathway through 6-phosphate glucose and 1, 6-fructose diphosphate.

251 High intakes of fructose were related to elevated risks of colorectal and pancreatic cancers 252 (Goncalves et al., 2019, Aune et al., 2012). The elevation of lactic acid and fructose in rats with 253 SCC and dysplasia suggested that those rats must consume more energy than normal rats.

Pyruvate and citric acid were higher in the serum of squamous cell carcinoma and

255 dysplasia rats. This possibly due to the need for more glycolysis to meet the increased energy 256 requirements of the cell, which was associated with accelerated anabolic metabolism. Pyruvate 257 can be converted to acetyl-CoA in the tricarboxylic acid (TCA) cycle, upon which it is 258 transaminated to alanine, or becomes lactate, particularly under hypoxic conditions (Raimundo et 259 al., 2011). Citric acid, an originate product in the TCA cycle, was elevated in rats with SCC and 260 dysplasia. Citric acid in human lung cancer was evaluated and compared with healthy controls 261 (Faubert et al., 2017, Hensley et al., 2016). The formation of citrate from acetyl-CoA and 262 oxaloacetate permits a new round of TCA cycling, generating high energy electrons, CO2, and 263 carbon skeletons that can be used for biosynthesis or anaplerosis. Citrate itself can be extruded 
264 into the cytosol and converted to acetyl-CoA by ATP citrate lyase (ACLY) for fatty acid synthesis 265 and the generation of biomembranes (Benjamin et al., 2012).

266 Extracellular high concentrations of pyruvate and citric acid support anabolism through the 267 fumarate-malate-citric acid pathway, resulting in the production of fatty acids and other 268 biochemical precursors in the carcinogenic process. The addition of pyruvate and citric acid can 269 also be used as chest substrates for the TCA cycle to increase energy production.

270 The levels of glycerol, hexadecanoic acid, palmitoylcarnitine, demissidine and 1271 hexadecyl-2-acetyl-glycero-3-phosphocholine in rats with SCC and dysplasia were significantly 272 different than those of the normal group. Those data implicate deregulated lipid biosynthesis in 273 cancer development. Dysregulated lipid metabolism and heightened de novo lipogenesis are 274 established hallmarks of cancer (Glaysher, 2013, Cheng et al., 2018). Tumor cells are 275 characterized by elevated fatty acid synthesis. Tumor cells synthesize fatty acids for the purposes 276 of membrane synthesis and for the generation of lipid signaling molecules to fuel cell proliferation 277 and cancer malignancy.

278 Significantly higher levels of glycine were observed in the model group compared to 279 controls. Glycine is the precursor for the biosynthesis of proteins, purine, and glutathione. In 280 addition, glycine is converted into sarcosine ( $\mathrm{N}$-methylglycine) by glycine $\mathrm{N}$-methyltransferase, 281 and conversely, sarcosine can be converted into glycine by sarcosine dehydrogenase (Locasale, 282 2013, Li et al., 2013). In recent years, tumor metabolomics studies have shown that glycine 283 metabolism is related to tumor cell proliferation. In the investigation of NCI-60 cell line plate, the 284 uptake and release rates of more than 200 metabolites were measured. Startlingly, lactic acid 285 production and glucose uptake (i.e., the Warburg effect) had nothing to do with cell proliferation.

286 After correlating individual metabolic fluences with cell proliferation, we found that glycine uptake 287 was most closely related to cancer cell proliferation. Isotopic tracers indicated that the cleavage 288 of glycine in the medium was involved in its catabolism, a pathway that has also been shown to 289 be necessary for rapid cell division (Jain et al., 2012). 
291 or uniquely expressed metabolites (Knobloch et al., 2012). These metabolites were mainly related

292 to Glycolysis and AMPK pathways. Along with targeting metabolic pathways, a comprehensive 293 analysis of their multiple sets of data further confirmed the array of molecular pathways regulated

294 by BRB phytochemicals. However, we did not use an independent method to verify our results.

295 In the process of chemoprevention of oral cancer, the characteristics of metabolites need to be

296 further identified and verified, which will provide more insights for the development of chemical 297 strategies in cancer prevention and treatment.

298

299 Conclusion:

300 In summary, we discovered a significant metabolic transformation in an 4NQO-induced

301 oral carcinogenesis model. The metabolic shift was characterized by an increase in 302 glycolysis and a deficiency in the TCA cycle. Other metabolic processes, including nucleic 303 acid and lipid biosynthesis, were also enhanced as part of cancer-associated metabolic 304 reprogramming. Our GC-MS metabolic analysis yielded a PLS-DA model, which showed

305 a good separation between the model group and the control groups.

306

307 Acknowledgements:

308 This study was supported by the Youth Project of the National Natural Science Foundation of 309 China (No. 30700944), and a grant from the Shanghai Municipal Health Bureau (ZHYY310 ZXYJHZX-201612).

311

\section{Disclosure:}

313 The author reports no conflicts of interest in this work. 


\section{References:}

317 Armitage EG \& Ciborowski M (2017). Applications of Metabolomics in Cancer Studies. Adv

$318 \quad$ Exp Med Biol 965: 209-234.

319 Aune D, Chan DS, Vieira AR, Navarro RD, Vieira R, Greenwood DC, Cade JE, Burley VJ

320 \&Norat T (2012). Dietary fructose, carbohydrates, glycemic indices and pancreatic

321 cancer risk: a systematic review and meta-analysis of cohort studies. Ann Oncol 23:

$322 \quad 2536-46$.

323 Bathen TF, Sitter B, Sjobakk TE, Tessem MB \&Gribbestad IS (2010). Magnetic resonance

324 metabolomics of intact tissue: a biotechnological tool in cancer diagnostics and treatment

325 evaluation. Cancer Res 70: 6692-6.

326 Beger RD (2013). A review of applications of metabolomics in cancer. Metabolites 3: 552-74.

327 Benjamin DI, Cravatt BF \& Nomura DK (2012). Global profiling strategies for mapping

328 dysregulated metabolic pathways in cancer. Cell Metab 16: 565-77.

329 Blekherman G, Laubenbacher R, Cortes DF, Mendes P, Torti FM, Akman S, Torti SV \&

330 Shulaev V (2011). Bioinformatics tools for cancer metabolomics. Metabolomics 7: 329-

$331 \quad 343$.

332 Chen T, Xie G, Wang X, Fan J, Qiu Y, Zheng X, Qi X, Cao Y, Su M, Wang X, Xu LX, Yen Y,

333 Liu P \& Jia W (2011). Serum and urine metabolite profiling reveals potential biomarkers

334 of human hepatocellular carcinoma. Mol Cell Proteomics 10: M110.004945.

335 Cheng C, Geng F, Cheng X \& Guo D (2018). Lipid metabolism reprogramming and its potential $336 \quad$ targets in cancer. Cancer Commun (Lond) 38: 27.

337 Choi SY, Collins CC, Gout PW \& Wang Y (2013). Cancer-generated lactic acid: a regulatory,

338 immunosuppressive metabolite? J Pathol 230: 350-5.

339 Dart A (2016). Tumour metabolism: Lactic acid: not just a waste product? Nat Rev Cancer 16:

340 676-677.

341 Faubert B, Li KY, Cai L, Hensley CT, Kim J, Zacharias LG, Yang C, Do QN, Doucette S, 
343

344

345

346

347

348

349

350

351

352

353

354

355

356

357

358

359

360

361

362

363

364

365

366

367

368

369

Lenkinski RE, Malloy CR, Wachsmann JW, Young JD, Kernstine K \& DeBerardinis RJ (2017). Lactate Metabolism in Human Lung Tumors. Cell 171: 358-371.e9.

Glaysher J (2013). Lipid metabolism and cancer. Curr Opin Lipidol 24: 530-1.

Goncalves MD, Lu C, Tutnauer J, Hartman TE, Hwang SK, Murphy CJ, Pauli C, Morris R, Taylor S, Bosch K, Yang S, Wang Y, Van Riper J, Lekaye HC, Roper J, Kim Y, Chen Q, Gross SS, Rhee KY, Cantley LC \& Yun J (2019). High-fructose corn syrup enhances intestinal tumor growth in mice. Science 363: 1345-1349.

Hensley CT, Faubert B, Yuan Q, Lev-Cohain N, Jin E, Kim J, Jiang L, Ko B, Skelton R, Loudat L, Wodzak M, Klimko C, McMillan E, Butt Y, Ni M, Oliver D, Torrealba J, Malloy CR, Kernstine K, Lenkinski RE \& DeBerardinis RJ (2016). Metabolic Heterogeneity in Human Lung Tumors. Cell 164: 681-94.

Jain M, Nilsson R, Sharma S, Madhusudhan N, Kitami T, Souza AL, Kafri R, Kirschner MW, Clish CB \& Mootha VK (2012). Metabolite profiling identifies a key role for glycine in rapid cancer cell proliferation. Science 336: 1040-4.

Kanojia D \& Vaidya MM (2006). 4-nitroquinoline-1-oxide induced experimental oral carcinogenesis. Oral Oncol 42: 655-67.

Kaushik AK, DeBerardinis RJ (2018).Applications of metabolomics to study cancer metabolism.Biochim Biophys Acta Rev Cancer: 1870:2-14.

Kim KB, Yang JY, Kwack SJ, Park KL, Kim HS, Ryu DH, Kim YJ, Hwang GS \& Lee BM (2010). Toxicometabolomics of urinary biomarkers for human gastric cancer in a mouse model. J Toxicol Environ Health A 73: 1420-30.

Knobloch TJ, Ryan NM, Bruschweiler-Li L, Wang C, Bernier MC, Somogyi A, Yan PS, Cooperstone JL, Mo X, Brüschweiler RP, Weghorst CM\& Oghumu S. (2019). Metabolic Regulation of Glycolysis and AMP Activated Protein Kinase Pathways during Black Raspberry-Mediated Oral Cancer Chemoprevention Metabolites: 9:140.

Kramer IR, Lucas RB, Pindborg JJ \& Sobin LH (1978). Definition of leukoplakia and related lesions: an aid to studies on oral precancer. Oral Surg Oral Med Oral Pathol 46: 518-39. 
370 Krishna RS, Mejia G, Roberts-Thomson K \& Logan R (2013). Epidemiology of oral cancer in 371 Asia in the past decade--an update (2000-2012). Asian Pac J Cancer Prev 14: 5567-77.

372 Leininger JR \& Jokinen MP (1994). Tumours of the oral cavity, pharynx, oesophagus and 373 stomach. IARC Sci Publ: 167-93.

374 Li Q, Lambrechts MJ, Zhang Q, Liu S, Ge D, Yin R, Xi M \& You Z (2013). Glyphosate and 375 AMPA inhibit cancer cell growth through inhibiting intracellular glycine synthesis. Drug Des Devel Ther 7: 635-43.

Locasale JW (2013). Serine, glycine and one-carbon units: cancer metabolism in full circle. Nat Rev Cancer 13: 572-83.

Markuszewski MJ, Struck W, Waszczuk-Jankowska M \& Kaliszan R (2010). Metabolomic approach for determination of urinary nucleosides as potential tumor markers using electromigration techniques. Electrophoresis 31: 2300-10.

Ninomiya T, Nagata M, Hata J, Hirakawa Y, Ozawa M, Yoshida D, Ohara T, Kishimoto H, Mukai N, Fukuhara M, Kitazono T \& Kiyohara Y (2013). Association between ratio of serum eicosapentaenoic acid to arachidonic acid and risk of cardiovascular disease: the Hisayama Study. Atherosclerosis 231: 261-7.

Ohashi T, Aoki M, Tomita H, Akazawa T, Sato K, Kuze B, Mizuta K, Hara A, Nagaoka H, Inoue N \& Ito Y (2017). M2-like macrophage polarization in high lactic acid-producing

Raimundo N, Baysal BE \& Shadel GS (2011). Revisiting the TCA cycle: signaling to tumor

Rivera C (2015). Essentials of oral cancer. Int J Clin Exp Pathol 8: 11884-94.

Sakai M, Kakutani S, Horikawa C, Tokuda H, Kawashima H, Shibata H, Okubo H \& Sasaki S (2012). Arachidonic acid and cancer risk: a systematic review of observational studies. BMC Cancer 12: 606. 
397 Tang XH, Knudsen B, Bemis D, Tickoo S \& Gudas LJ (2004). Oral cavity and esophageal carcinogenesis modeled in carcinogen-treated mice. Clin Cancer Res 10: 301-13.

399

400

401

402

403

404

405

406

407

408

409

411

412

413

414

415

416

417

418

419

420

421

WARBURG O (1956). On the origin of cancer cells. Science 123: 309-14.

Warnakulasuriya S \& Ariyawardana A (2016). Malignant transformation of oral leukoplakia: a systematic review of observational studies. J Oral Pathol Med 45: 155-66.

Wei J, Xie G, Ge S, Qiu Y, Liu W, Lu A, Chen T, Li H, Zhou Z \& Jia W (2012). Metabolic transformation of DMBA-induced carcinogenesis and inhibitory effect of salvianolic acid b and breviscapine treatment. J Proteome Res 11: 1302-16.

Wen H, Yoo SS, Kang J, Kim HG, Park JS, Jeong S, Lee JI, Kwon HN, Kang S, Lee DH \& Park S (2010). A new NMR-based metabolomics approach for the diagnosis of biliary tract cancer. J Hepatol 52: 228-33.

Wu H, Xue R, Tang Z, Deng C, Liu T, Zeng H, Sun Y \& Shen X (2010). Metabolomic investigation of gastric cancer tissue using gas chromatography/mass spectrometry. Anal Bioanal Chem 396: 1385-95.

Yarla NS, Bishayee A, Sethi G, Reddanna P, Kalle AM, Dhananjaya BL, Dowluru KS, Chintala R \& Duddukuri GR (2016). Targeting arachidonic acid pathway by natural products for cancer prevention and therapy. Semin Cancer Biol 40-41: 48-81. 


\section{Figure Legends}

426 Figure 1.Histopathological images from 4NQO-induced oral lesions $(\times 200)$.

427 (A)Normal squamous epithelium of the tongue

428 (B) Mild epithelial dysplasia

429 (C) Moderate epithelial dysplasia

430 (D) Severe epithelial dysplasia

431 (E) Squamous cell carcinoma (SCC)

432 Figure 2.Score plot for the PLS-DA model of the GC-MS spectral data from the model group and 433 the healthy control group.

434 Table 1. Histological changes in rat tongue by 4NQO treatment

435 Table 2. List of Identified Differential Metabolites between Model Group and Healthy Controls

436 aMetabolites verified by reference compounds, other were directly obtained from library 437 searching.

438 bFold change was calculated from the arithmetic mean values of each group. Fold change with 439 a positive value indicates a relatively higher concentration present in model group while a negative 440 value means a relatively lower concentration as compared to the healthy controls.

$441{ }^{\mathbf{c}}$ Variable importance in the projection (VIP) was obtained from PLS-DA with a threshold of 4421.0.

$443 \quad{ }^{\mathrm{d}} \mathrm{p}$ value was calculated from student $\mathrm{t}$ test. 


\section{Figure 1}

Histopathological images from 4NQO-induced oral lesions $(\times 200)$.

(A)Normal squamous epithelium of the tongue

(B) Mild epithelial dysplasia

(C) Moderate epithelial dysplasia

(D) Severe epithelial dysplasia

(E) Squamous cell carcinoma (SCC)

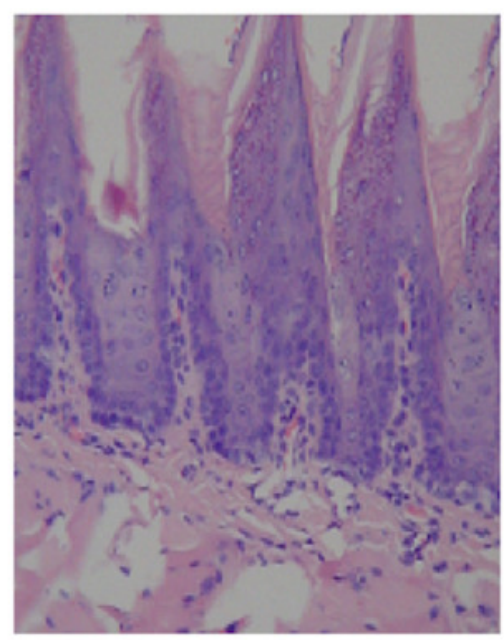

A
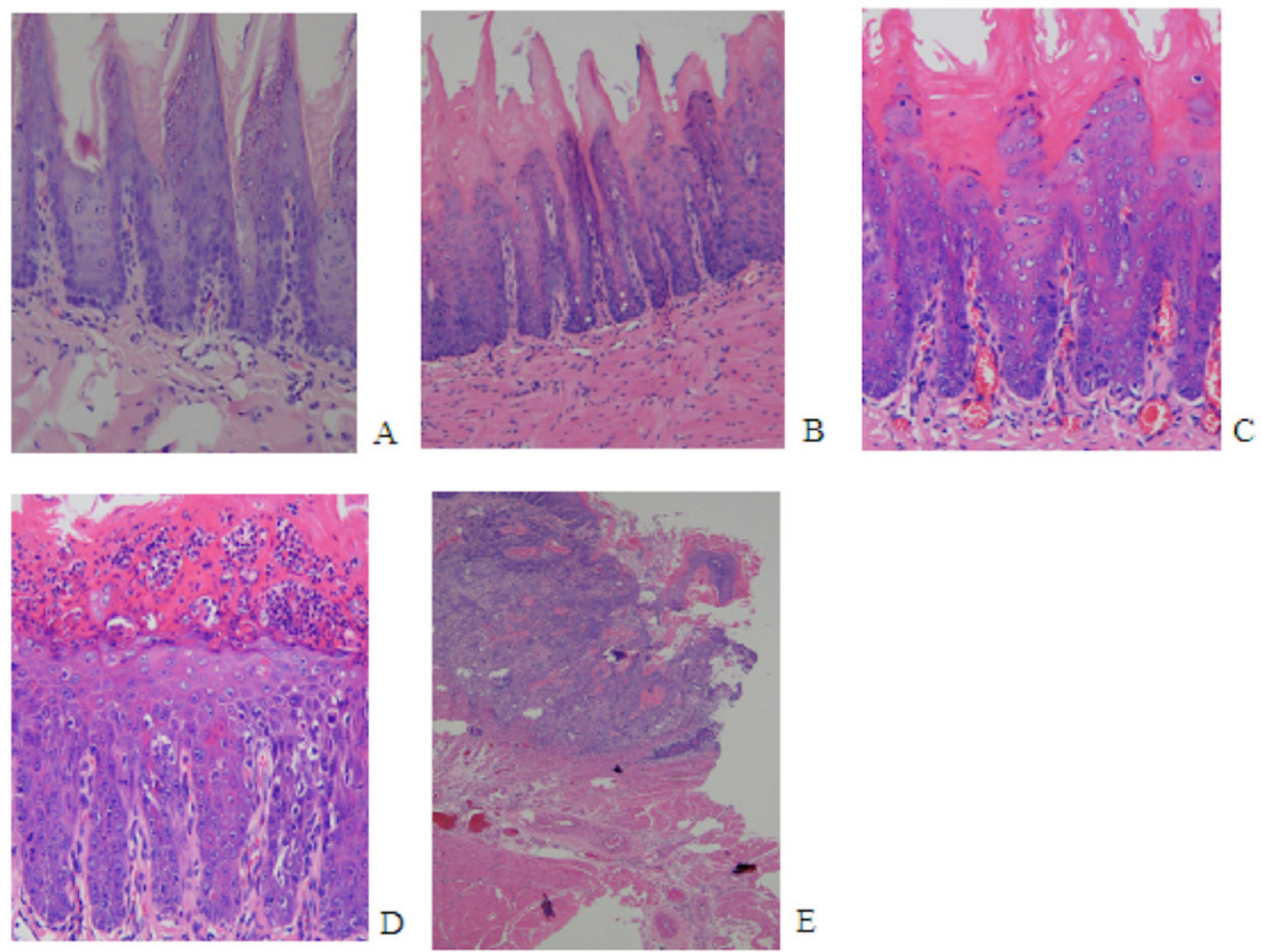
Figure 2

Score plot for the PLS-DA model of the GC-MS spectral data from the model group and the healthy control group.
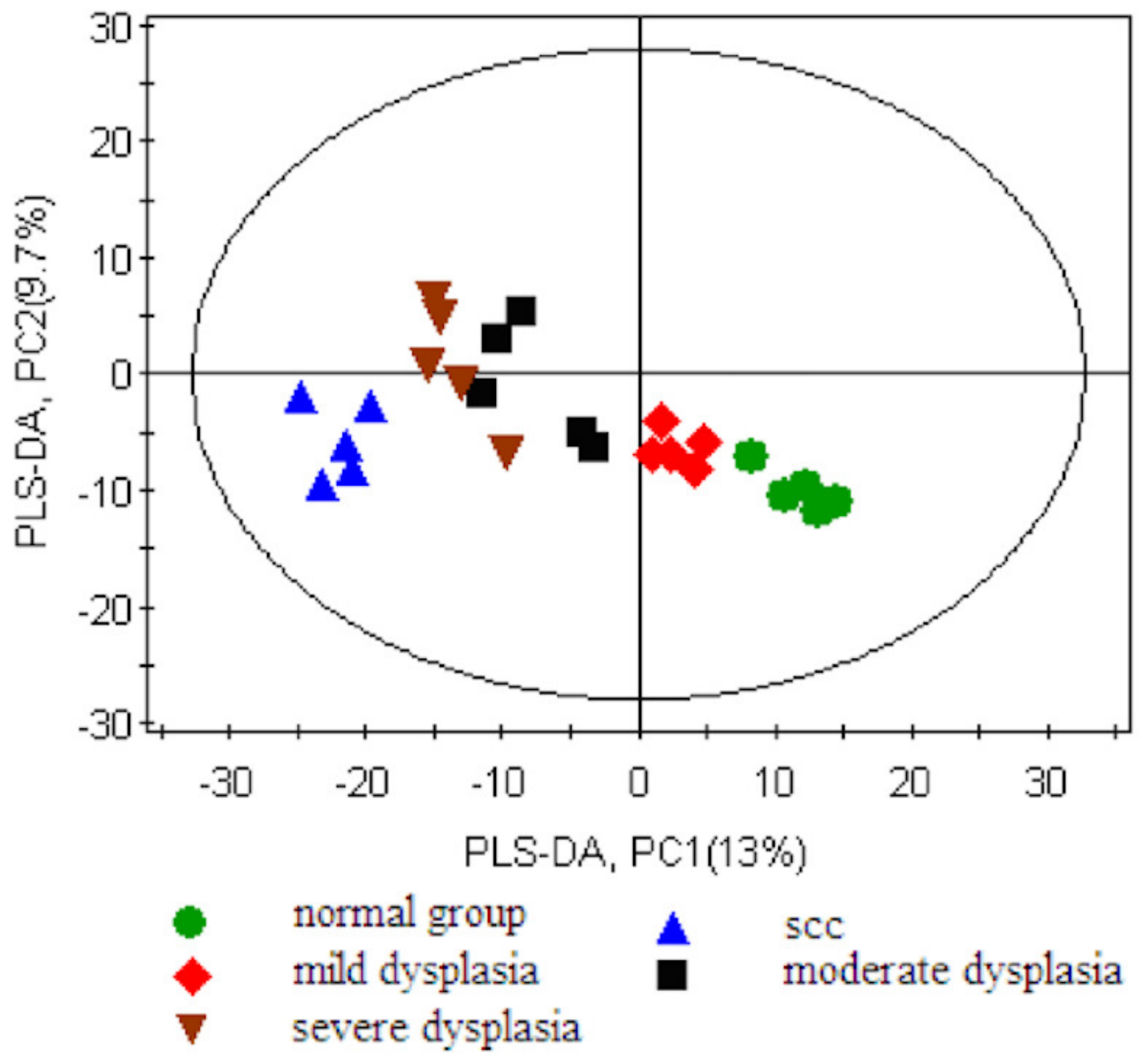


\section{Table $\mathbf{1}$ (on next page)}

Histological changes in rat tongue by $4 \mathrm{NQO}$ treatment 
1 Table 1. Histological changes in rat tongue by 4NQO treatment

\begin{tabular}{ccccccc}
\hline $\begin{array}{c}\text { group } \\
\text { (week) }\end{array}$ & $\begin{array}{c}\text { No. of } \\
\text { normal }\end{array}$ & $\begin{array}{c}\text { mild } \\
\text { epithelial } \\
\text { dysplasia }\end{array}$ & $\begin{array}{c}\text { moderate } \\
\text { epithelial } \\
\text { dysplasia }\end{array}$ & $\begin{array}{c}\text { Severe } \\
\text { epithelial } \\
\text { dysplasia }\end{array}$ & $\begin{array}{c}\text { No. of } \\
\text { SCC }\end{array}$ & $\begin{array}{c}\text { Incidence } \\
(\%)\end{array}$ \\
\hline control & 5 & 0 & 0 & 0 & 0 & 0 \\
9week & 0 & 4 & 3 & 0 & 0 & 0 \\
13week & 0 & 1 & 5 & 1 & 0 & 0 \\
20week & 0 & 0 & 5 & 0 & 0 & 0 \\
24week & 0 & 0 & 0 & 2 & 4 & 33.3 \\
32week & 0 & 0 & 0 & 0 & 8 & 100 \\
\hline
\end{tabular}




\section{Table 2 (on next page)}

\section{List of Identified Differential Metabolites between Model Group and Healthy Controls}

a Metabolites verified by reference compounds, other were directly obtained from library searching.

${ }^{\mathbf{b}}$ Fold change was calculated from the arithmetic mean values of each group. Fold change with a positive value indicates a relatively higher concentration present in model group while a negative value means a relatively lower concentration as compared to the healthy controls.

c Variable importance in the projection (VIP) was obtained from PLS-DA with a threshold of 1.0.

${ }^{d} \mathrm{p}$ value was calculated from student t test. 
1 Table 2. List of Identified Differential Metabolites between Model Group and Healthy Controls

\begin{tabular}{ccccc}
\hline No & metabolite $^{\mathrm{a}}$ & $\mathrm{FC}^{\mathrm{b}}$ (model/control) & $\mathrm{VIP}^{\mathrm{c}}$ & $p^{\mathrm{d}}$ \\
\hline 1 & PGF2alpha dimethyl amide & -1.37 & 1.63 & 0.0138 \\
2 & 3',5'-Cyclic dGMP & -1.03 & 1.42 & 0.042 \\
3 & lactic acid & 2.84 & 1.55 & 0.0036 \\
4 & Palmitoylcarnitine & 1.04 & 1.53 & 0.0243 \\
5 & Demissidine & 1.48 & 1.55 & 0.0383 \\
6 & 16a-Hydroxydydrogesterone & 1.61 & 1.54 & 0.0069 \\
7 & arachidonic acid & 2.21 & 1.91 & 0.0028 \\
8 & Deoxyuridine monophosphate & 1.15 & 1.79 & 0.0159 \\
& (dUMP) & & & \\
9 & oleamide & 1.78 & 1.7 & 0.0078 \\
10 & citric acid & 1.85 & 2.14 & 0.0174 \\
11 & 1-hexadecyl-2-acetyl-glycero- & -1.25 & 2.05 & 0.0162 \\
& 3-phosphocholine & & & \\
12 & glycerol & 1.89 & 1.65 & 0.022 \\
13 & glycine & 1.497 & 1.64 & 0.0056 \\
14 & hexadecanoic acid & 1.518 & 1.52 & 0.0101 \\
15 & pyruvate & 1.832 & 1.66 & 0.00123 \\
16 & eicosatetraenoic acid & 1.18 & 1.84 & 0.0239 \\
\hline
\end{tabular}

2 